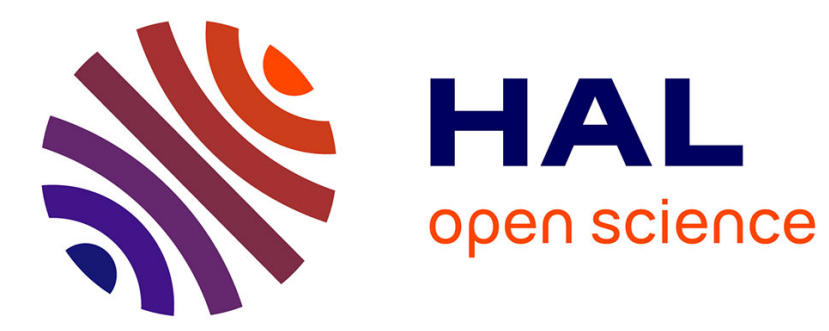

\title{
Analysis of scale invariance property applying homogeneity
}

\author{
Emmanuel Bernuau, Denis Efimov, Wilfrid Perruquetti
}

\section{To cite this version:}

Emmanuel Bernuau, Denis Efimov, Wilfrid Perruquetti. Analysis of scale invariance property applying homogeneity. 19th IFAC World Congress 2014, Aug 2014, Cape Town, South Africa. hal-00964081

\section{HAL Id: hal-00964081 \\ https://hal.inria.fr/hal-00964081}

Submitted on 26 Mar 2014

HAL is a multi-disciplinary open access archive for the deposit and dissemination of scientific research documents, whether they are published or not. The documents may come from teaching and research institutions in France or abroad, or from public or private research centers.
L'archive ouverte pluridisciplinaire HAL, est destinée au dépôt et à la diffusion de documents scientifiques de niveau recherche, publiés ou non, émanant des établissements d'enseignement et de recherche français ou étrangers, des laboratoires publics ou privés. 


\title{
Analysis of scale invariance property applying homogeneity
}

\author{
Emmanuel Bernuau ${ }^{* *}$ Denis Efimov ${ }^{*, * * *, * * * *}$ \\ Wilfrid Perruquetti ${ }^{*, * * *}$ \\ *Non-A team @ Inria, Parc Scientifique de la Haute Borne, 40 avenue \\ Halley, 59650 Villeneuve d'Ascq, France \\ ** Faculty of Engineering, University of Pisa, Largo Lucio Lazzarino 1, \\ 56122 Pisa, Italy \\ *** LAGIS (UMR-CNRS 8146), Ecole Centrale de Lille, BP 48, Cité \\ Scientifique, 59651 Villeneuve-d'Ascq, France \\ **** Department of Control Systems and Informatics, National \\ Research University ITMO, 49 av. Kronverkskiy, 197101 Saint \\ Petersburg, Russia
}

\begin{abstract}
The problem of scalability of trajectories in homogeneous and locally homogeneous systems is considered. It is shown that the homogeneous systems have scalability property, and locally homogeneous systems possess this property approximately. The issue of closeness of trajectories for the system and its local homogeneous approximation is investigated. The notions of scale invariance and fold change detection are also studied. The results are illustrated by a cascade system analysis.
\end{abstract}

\section{INTRODUCTION}

Many biological systems demonstrate an adaptation ability, i.e. they are capable to maintain the levels of essential variables in the admissible bounds in the presence of wide deviations of the range of inputs Alon [2006]. In some cases they even demonstrate a more strong property called (approximate) scale invariance, when the output of the system becomes (almost) the same after the input scaling Shoval et al. [2011], Skataric and Sontag [2012], Hamadeh et al. [2013]. Constructive conditions of presence of this property are demanded in systems biology.

The conditions of scale invariance obtained in Shoval et al. [2011], Skataric and Sontag [2012], Hamadeh et al. [2013] are based on some kind of symmetry verification and linearization. It is shown that if a system is symmetric, then under some additional minor restrictions it has the scale invariance property. Roughly speaking a system admits the scale invariance property if its transient behavior stays approximately the same when the input signal level is scaled. Such a type of dynamical behavior is a kind of symmetry. Homogeneity is a concept, which has been introduced in order to study the influence of symmetry on behavior (stability, robustness etc.) of solutions of nonlinear systems Bacciotti and Rosier [2005], Bhat and Bernstein [2005]. Homogeneity is also a kind of symmetry. Verification of the presence of this type of symmetry is rather simple since it is based on arithmetic operation and/or algebraic tests.

The objective of this note is to investigate the scale invariance property using the homogeneity framework. First, it is shown that the results on the presence of scale

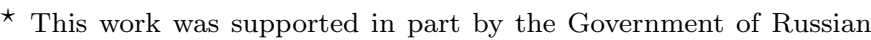
Federation (Grant 074-U01) and the Ministry of Education and Science of Russian Federation (Project 14.Z50.31.0031).
}

invariance under a generic symmetry of the differential equations describing the system can be easily re-casted in the homogeneity framework. Second, it is shown how local homogeneity implies approximate scale invariance in the system. Several examples demonstrating efficiency of the homogeneity application for scaling analysis are given.

The outline of the presentation is as follows. In Section 2 some preliminary results and definitions of homogeneity and scale invariance are presented. In Section 3 the link between homogeneity and scalability is established. The relation between approximate scalability and local homogeneity is investigated in Section 4.

\section{PRELIMINARIES}

\subsection{Notation}

In this paper, the following notation will be used.

- We denote $\mathbb{R}_{+}=\{x \in \mathbb{R}: x \geq 0\}$ and $\mathbb{R}_{+}^{*}=\mathbb{R}_{+} \backslash\{0\}$. If $E \subset \mathbb{R}$ we set $E_{+}=E \cap \mathbb{R}_{+}$and $E_{+}^{*}=E \cap \mathbb{R}_{+}^{*}$. We denote $\mathbb{N}=\mathbb{Z}_{+}$and $\mathbb{N}^{*}=\mathbb{Z}_{+} \cap \mathbb{R}_{+}^{*}$.

- If $n \in \mathbb{N}^{*}$, we denote $\operatorname{Lip}\left(\mathbb{R}^{n}\right)$ the set of locally Lipschitz continuous vector fields on $\mathbb{R}^{n}$.

- Denote $\Psi$ the flow of a $F \in \operatorname{Lip}\left(\mathbb{R}^{n}\right)$, then $\Psi^{t}(x)$ represents the state of the trajectory of $F$ at time $t$ with the initial condition $x$, i.e. $\Psi^{0}(x)=x$. We denote $\frac{d}{d t} \Psi^{t}(x)$ the time derivative of this curve $\left(\frac{d}{d t} \Psi^{t}(x)=\right.$ $F\left(\Psi^{t}(x)\right)$ by definition) and $d_{x} \Psi^{t}$ the differential at $x$ of the diffeomorphism $x \mapsto \Psi^{t}(x), t$ being fixed.

- If $f$ is a $C^{1}$ vector field on $\mathbb{R}^{n}$, we denote $\operatorname{Jac} f(x)$ the Jacobian matrix of $f$ taken at the point $x \in \mathbb{R}^{n}$.

- For two $C^{1}$ vector fields $f, g: \mathbb{R}^{n} \rightarrow \mathbb{R}^{n}$, denote their Lie brackets $[f, g]=\frac{\partial g}{\partial x} f(x)-\frac{\partial f}{\partial x} g(x)$. For a $C^{1}$ vector field $f: \mathbb{R}^{n} \rightarrow \mathbb{R}^{n}$ and a $C^{1}$ function $V: \mathbb{R}^{n} \rightarrow \mathbb{R}$ the 
Lie derivative of $V$ along the vector field $f$ is defined as $\mathcal{L}_{f} V=\frac{\partial V}{\partial x} f(x)$.

- Given matrices $\mathcal{A}_{k} \in \mathbb{R}^{n \times n}, 1 \leq k \leq K, K \in \mathbb{N}^{*}$, define the convex hull of these matrices

$$
\begin{gathered}
C o\left(\mathcal{A}_{1}, \ldots, \mathcal{A}_{K}\right)=\left\{A \in \mathbb{R}^{n \times n}: A=\sum_{k=1}^{K} \alpha_{k} \mathcal{A}_{k},\right. \\
\left.\alpha_{k} \in[0,1], \sum_{k=1}^{K} \alpha_{k}=1\right\} .
\end{gathered}
$$

- For a symmetric matrix $A \in \mathbb{R}^{n \times n}$ denote $\lambda_{\max }(A)$ and $\lambda_{\min }(A)$ the biggest and smallest eigenvalues respectively.

- If $A$ and $B$ are two subsets of $\mathbb{R}^{n}$, we denote $A+$ $B=\{a+b, a \in A, b \in B\}$.

- For a measurable function $u: \mathbb{R}_{+} \rightarrow \mathbb{R}^{m}$ define the norms $\|u\|_{\mathcal{L}^{1}[a, b]}=\int_{a}^{b}|u(t)| d t$ and $\|u\|_{\mathcal{L}^{\infty}[a, b]}=$ $\sup _{t \in[a, b]}|u(t)|$ for any $0 \leq a \leq b \leq+\infty$.

\subsection{Homogeneity concepts}

In order to use the symmetry that some systems present, the weighted homogeneity has been introduced Zubov [1958].

Definition 1. Set $\mathbf{r}=\left(r_{1}, \ldots, r_{n}\right)$ with $r_{i} \in \mathbb{R}_{+}^{*}$. A dilation associated to the generalized weight $\mathbf{r}$ is a group action: $\lambda \in \mathbb{R}_{+}^{*} \mapsto \Lambda_{\mathbf{r}}(\lambda)$ with $\Lambda_{\mathbf{r}}(\lambda): x \mapsto\left(\lambda^{r_{1}} x_{1}, \ldots, \lambda^{r_{n}} x_{n}\right)$. The mapping $\Lambda_{\mathbf{r}}(\lambda)$ is often simply denoted by $\Lambda_{\mathbf{r}}$ (the $\lambda$ is omitted).

Definition 2. A function $V: \mathbb{R}^{n} \rightarrow \mathbb{R}$ (resp. a vector field $F: \mathbb{R}^{n} \rightarrow \mathbb{R}^{n}$ ) is said to be $\mathbf{r}$-homogeneous of degree $d$ iff for all $\lambda>0$ and for all $x \in \mathbb{R}^{n}$ we have $\lambda^{-d} V\left(\Lambda_{\mathbf{r}} x\right)=V(x)$ (resp. $\left.\lambda^{-d} \Lambda_{\mathbf{r}}^{-1} F\left(\Lambda_{\mathbf{r}} x\right)=F(x)\right)$.

Note that a vector field $F=\left(F_{1}, \ldots, F_{n}\right)$ is $\mathbf{r}$-homogeneous of degree $d$ iff for each $i \in\{1, \ldots, n\}$, the function $F_{i}$ is r-homogeneous of degree $r_{i}+d$. The homogeneous norm can be defined as follows

$$
N(x)=\left(\sum_{i=1}^{n}\left|x_{i}\right|^{\rho / r_{i}}\right)^{1 / \rho}, \rho \geq \max _{1 \leq i \leq n} r_{i},
$$

this norm is $\mathbf{r}$-homogeneous.

Being a kind of symmetry, the homogeneity property should be invariant under a change of coordinates. But this is not true for the weighted homogeneity. This motivates for a geometric, coordinate-free definition of homogeneity Kawski [1995].

Definition 3. A vector field $\nu: \mathbb{R}^{n} \rightarrow \mathbb{R}^{n}$ is said to be Euler if $\nu$ is $C^{1}$ and the origin is Globally Asymptotically Stable (GAS) for $-\nu$. In the sequel, we will restrict ourselves to complete Euler vector fields. We will always write $\Phi$ the flow of $\nu$.

Definition 4. Let $\nu$ be an Euler vector field. A function $V$ is said to be $\nu$-homogeneous of degree $d$ iff for all $s \in \mathbb{R}$ we have:

$$
e^{-d s} V\left(\Phi^{s}(x)\right)=V(x) .
$$

A vector field $F$ is said to be $\nu$-homogeneous of degree $d$ iff for all $s \in \mathbb{R}$ we have:

$$
e^{-d s}\left(d_{x} \Phi^{s}\right)^{-1} F\left(\Phi^{s}(x)\right)=F(x) .
$$

It is straightforward to check that considering the generalized weight $\mathbf{r}=\left(r_{1}, \ldots, r_{n}\right)$, with $r_{i}>0$ and setting $\nu(x)=r_{1} x_{1} \frac{\partial}{\partial x_{1}}+\ldots+r_{n} x_{n} \frac{\partial}{\partial x_{n}}$ in a fixed basis, a function or a vector field will be $\mathbf{r}$-homogeneous iff it is $\nu$ homogeneous with the same degree.

Proposition 5. A $C^{1}$ function $V$ is $\nu$-homogeneous with degree $d$ iff $\mathcal{L}_{\nu} V=d V$. A $C^{1}$ vector field $F$ is $\nu$ homogeneous with degree $d$ iff $[\nu, F]=d F$.

The main feature of homogeneous systems (i.e. systems defined by a homogeneous vector field) is the following proposition about a kind of symmetry of trajectories, or a commutation property of flows Kawski [1995].

Proposition 6. Let $F \in \operatorname{Lip}\left(\mathbb{R}^{n}\right)$ and denote by $\Psi$ its flow. Then $F$ is $\nu$-homogeneous of degree $d$ iff

$$
\Phi^{s} \circ \Psi^{e^{d s} t}=\Psi^{t} \circ \Phi^{s} \quad \forall s, t \in \mathbb{R} .
$$

Due to this result, if a homogeneous system is locally asymptotically stable (attractive), then it is GAS.

Example 1. Consider the following scalar system:

$$
\dot{x}=-\frac{a x}{b+|x|},
$$

where $a>0$ and $b>0$ are parameters. Obviously, this system is not homogeneous with respect to conventional Definition 2. However, it is straightforward to verify using the last proposition that it is homogeneous in the sense of Definition 4 with $d=1$ for $\nu(x)=b^{-1} x(b+|x|)$.

\subsection{Scalability and scale invariance}

In this section, $n, m \in \mathbb{N}^{*}$. Consider now a system

$$
\begin{aligned}
& \dot{x}=f(x, u), \\
& y=h(x),
\end{aligned}
$$

where $x \in \mathbb{R}^{n}$ is the state, $u \in \mathbb{U} \subset \mathbb{R}^{m}$ is the input and $y \in \mathbb{R}$ is the output, $f: \mathbb{R}^{n+m} \rightarrow \mathbb{R}^{n}$ is locally Lipschitz continuous. The set $\mathbb{U}$ is the set of admissible inputs, namely a subset of the measurable inputs from $\mathbb{R}$ to $\mathbb{R}^{m}$. We assume that $\mathbb{U}$ is invariant with respect to a positive scaling. We assume that for any $\bar{u} \in \mathbb{R}^{m}$, there exists a unique $\sigma(\bar{u}) \in \mathbb{R}^{n}$ such that $f(\sigma(\bar{u}), \bar{u})=0$. Let us denote by $x\left(t, x_{0}, u\right)$ the current state of the system under input $u$ and with initial condition $x_{0}$. Finally, we denote $y\left(t, x_{0}, u\right)=h\left(x\left(t, x_{0}, u\right)\right)$.

Following Shoval et al. [2011], Skataric and Sontag [2012] let us introduce the main properties under consideration in this note.

Definition 7. The system (2) is said to be positive Scalable $(\mathrm{pS})$ with respect to the inputs from $\mathbb{U}$ if there exists a function $\beta: \mathbb{R}_{+}^{*} \rightarrow \mathbb{R}_{+}^{*}$ such that for any $\bar{u} \in \mathbb{R}^{m}$, any input $u \in \mathbb{U}$ and any $\lambda>0$ :

$$
y(t, \sigma(\lambda \bar{u}), \lambda u)=\beta(\lambda) y(t, \sigma(\bar{u}), u) \quad \forall t \geq 0 .
$$

It is worth to stress that comparing with Shoval et al. [2011], Skataric and Sontag [2012], Hamadeh et al. [2013], in this work only positive scaling $\lambda$ is considered, that is why the property is called pS. In Shoval et al. [2011] much more general $\mathcal{P}$-invariance property has been considered, where $\mathcal{P}$ is a set of $C^{0}$ input transformations, while in the present work only multiplication by a constant $\lambda$ is analyzed. 
In this definition the constant $\bar{u} \in \mathbb{R}^{m}$ represents the influence of inputs applied before the instant $t=0$, then the system starts its movement from the equilibrium $\sigma(\bar{u})$ corresponding to that input. The input $u \in \mathbb{U}$ is applied for $t \geq 0$ and the scalability property means that if all inputs have been scaled by a $\lambda>0$ (i.e. $\bar{u}$ and $u$ ), then the output of the system is scaled by $\beta(\lambda)$.

If this property is satisfied and the system is excited by two different inputs $u \in \mathbb{U}$ and $u^{\prime} \in \mathbb{U}$, then

$$
\frac{y(t, \sigma(\bar{u}), u)}{y\left(t, \sigma\left(\bar{u}^{\prime}\right), u^{\prime}\right)}=\frac{y(t, \sigma(\lambda \bar{u}), \lambda u)}{y\left(t, \sigma\left(\lambda \bar{u}^{\prime}\right), \lambda u^{\prime}\right)}
$$

for any $\lambda>0$, therefore the scaling factor $\lambda$ is unimportant and only the "ratio" $\frac{u}{u^{\prime}}$ has to be taken into account.

Definition 8. A pS system (2) with $\beta=1$ is said to admit a positive Fold Change Detection (pFCD) property.

A pFCD system (2) has exactly the same response for an input $u$ applied at the initial condition $\sigma(\bar{u})$ as for an input $\lambda u$ applied at the initial condition $\sigma(\lambda \bar{u})$.

\section{APPLICATION OF HOMOGENEITY}

In the work Shoval et al. [2011] the generic conditions, for a dynamical systems to have invariant response with respect to symmetries in $\mathcal{P}$, have been proposed (for the case $\beta=1$ ). Homogeneity is a particular kind of symmetry, thus in this section we reformulate and partially repeat the results of Shoval et al. [2011] using homogeneity theory. This development will be used in the next section for investigation of approximate scale invariance property based on the homogeneity framework.

We assume the following homogeneity properties for the system (2).

Assumption 1. There exists an Euler vector field $\nu_{1}$ : $\mathbb{R}^{n} \rightarrow \mathbb{R}^{n}$, whose flow is denoted by $\Phi_{1}$, such that $f\left(\Phi_{1}^{s}(x), e^{s} u\right)=d_{x} \Phi_{1}^{s} f(x, u)$.

The function $h$ is $\nu_{1}$-homogeneous with degree $p$.

Remark 9. Note that under these conditions, if for $u=$ 0 the system (2) is GAS, then (2) is Input-to-State Stable (ISS) Bernuau et al. [2013a,b, 2014] (in the case of coordinate-free homogeneity the ISS property depends on characteristics of $\nu$ ).

In this section, we will study the pS property of the system (2) under the class of locally bounded inputs, denoted by $\mathbb{U}$, but we will first look at this property for a smaller class of inputs, denoted by $\mathbb{U}$. Let us define this class.

Let $q \in \mathbb{N}_{+}^{*}$, for all $g \in \operatorname{Lip}\left(\mathbb{R}^{q}\right)$, we denote $\Xi_{g}$ the flow of $g$. A triple $\left(\phi, g, \xi_{0}\right)$, with a continuous $\phi: \mathbb{R}^{q} \rightarrow \mathbb{R}^{m}$, $g \in \operatorname{Lip}\left(\mathbb{R}^{q}\right)$ and $\xi_{0} \in \mathbb{R}^{q}$, is said to be an admissible $q-$ triple if there exists an Euler vector field $\nu_{2}: \mathbb{R}^{q} \rightarrow \mathbb{R}^{q}$ with flow $\Phi_{2}$ such that $g$ is $\nu_{2}$-homogeneous with degree 0 and $\phi \circ \Phi_{2}^{s}=e^{s} \phi$ for all $s \in \mathbb{R}$ (if $m=1$, then this property means that $\phi$ is $\nu_{2}$-homogeneous with degree 1 ).

We denote $\mathbb{T}_{q}$ the set of admissible $q$-triples. The set of admissible inputs $u$ we consider is given by:

$$
\begin{aligned}
\mathbb{U}=\{u: \mathbb{R} & \rightarrow \mathbb{R}^{m}: \exists q>0, \exists\left(\phi, g, \xi_{0}\right) \in \mathbb{T}_{q}: \\
u(t) & \left.=\phi\left(\Xi_{g}^{t}\left(\xi_{0}\right)\right)\right\} .
\end{aligned}
$$

Any triple $\left(\phi, g, \xi_{0}\right) \in \mathbb{T}_{q}$ such that $u(t)=\phi\left(\Xi_{g}^{t}\left(\xi_{0}\right)\right) \in \mathbb{U}$, we will call an associated $q$-triple for the corresponding $u \in \mathbb{U}$. Therefore, we will consider the class of inputs $\mathbb{U}$ generated by a homogeneous differential equation:

$$
\dot{\xi}(t)=g(\xi(t)), \quad u(t)=\phi(\xi(t))
$$

with the initial condition $\xi_{0} \in \mathbb{R}^{q}$. Thus the scaling property under consideration in this note can be interpreted as the output scalability for a consequent connection of two dynamical systems, when positive rescaling of initial conditions for the first system leads to a corresponding scaling of the output of the second system.

Proposition 10. The set $\mathbb{U}$ is a vector space.

All proofs are excluded due to the space limitations.

\subsection{Homogeneity conditions for $p S$ property}

For an initial condition $x_{0} \in \mathbb{R}^{n}$ and an input $u \in \mathbb{U}$, consider an associated $q$-triple $\left(\phi, g, \xi_{0}\right)$ and define:

$$
F\left(\begin{array}{l}
x \\
\xi
\end{array}\right)=\left(\begin{array}{c}
f(x, \phi(\xi)) \\
g(\xi)
\end{array}\right) .
$$

We denote $\Psi$ the flow of $F$. Denote $p_{1}: \mathbb{R}^{n} \times \mathbb{R}^{q} \rightarrow \mathbb{R}^{n}$ and $p_{2}: \mathbb{R}^{n} \times \mathbb{R}^{q} \rightarrow \mathbb{R}^{q}$ the first and second canonical projections respectively, then $y\left(t, x_{0}, u\right)=h\left(p_{1} \circ \Psi^{t}\left(x_{0}, \xi_{0}\right)\right)$.

Let us denote $\nu\left(\begin{array}{l}x \\ \xi\end{array}\right)=\left(\begin{array}{c}\nu_{1}(x) \\ \nu_{2}(\xi)\end{array}\right)$ and $\Phi$ the flow of $\nu$.

Lemma 11. If Assumption 1 is verified, the following properties hold:

1. The vector field $F$ is $\nu$-homogeneous with degree 0 .

2. Denote $\Phi_{1}^{s}=p_{1} \circ \Phi^{s}$, then $\Phi_{1}^{s}(\sigma(\bar{u}))=\sigma\left(e^{s} \bar{u}\right)$.

3. Denote $\Psi_{2}^{t}=p_{2} \circ \Psi^{t}$, then the mapping $\Psi_{2}$ is the flow of $g$ and $\Psi_{2}^{t} \circ \Phi_{2}^{s}=\Phi_{2}^{s} \circ \Psi_{2}^{t}$.

Theorem 12. Under Assumption 1, the system (2) is pS with respect to inputs from $\mathbb{U}$ and $\beta(\lambda)=\lambda^{p}$. Moreover, if $p=0$, then the system admits $\mathrm{pFCD}$ property.

Corollary 13. Under Assumption 1, the system (2) is pS with respect to polynomials inputs.

Theorem 14. Under Assumption 1, the system (2) is pS with respect to locally bounded inputs.

Lemma 15. Let $u$ be a bounded input defined on $[0, T]$. Suppose that there exists a sequence $\left(u_{n}\right)_{n>1}$ of bounded inputs converging to $u$ in $\mathcal{L}^{1}[0, T]$ (i.e. $\left\|u_{n}(t)-u(t)\right\|_{\mathcal{L}^{1}[0, T]}$ $\rightarrow 0$ when $n \rightarrow+\infty)$ such that $\left\|u_{n}\right\|_{\mathcal{L}^{\infty}[0, T]} \leq 2\|u\|_{\mathcal{L}^{\infty}[0, T]}$. Let $x$ be the trajectory of the system (2) with input $u$ and initial condition $x(0)=x_{0}$ and $x_{n}$ be the trajectory of the system (2) with input $u_{n}$ and initial condition $x_{n}(0)=x_{0}$. Then $x_{n}$ converges to $x$ uniformly on $[0, T]$.

\subsection{The case of constant inputs}

Let $\mathbb{U}$ be the class of constant inputs, in this case

$$
g(\xi)=0, \quad \phi(\xi)=\xi
$$

then $\nu_{2}(\xi)=\xi$. Following the result of Lemma 11, to verify Assumption 1 it is equivalent to check that the vector field $F(x, u)=\left[f(x, u)^{T} 0\right]^{T}$ is homogeneous for $\nu(x, u)=\left[\nu_{1}(x)^{T} u\right]^{T}$ with degree $d=0$.

Consider examples of such a case study.

Example 2. Consider a linear system with

$$
f(x, u)=A x+b u, y=c^{T} x,
$$


where $A$ is an invertible matrix of dimension $n \times n, b$ and $c$ are some vectors in $\mathbb{R}^{n}$. This system is (weighted) homogeneous with $d=0$ and $p=1$ and, hence, it admits the pS property with respect to constant inputs. For $y=\operatorname{sign}\left(c^{T} x\right)$ the system has pFCD property.

Example 3. Take

$$
f(x, u)=-\frac{a x}{1+|x|}+\frac{e^{-|x|}}{1+|x|} u, h(x)=e^{p|x|} x^{p},
$$

where $a>0, p>0$, then $F$ and $h$ are homogeneous for $\nu_{1}(x)=\frac{x}{1+|x|}$ with the degrees $d=0$ and $p$ respectively. Thus this nonlinear system is $\mathrm{pS}$ with respect to constant inputs.

\section{LOCAL HOMOGENEITY}

Previously we assumed that the vector field $F$ is homogeneous, which is a rather restrictive hypothesis. This assumption may be relaxed using local homogeneity Zubov [1958], Andrieu et al. [2008], Efimov and Perruquetti [2010].

Definition 16. Let $F_{0}$ be a vector field such that

$$
e^{-d s}\left(d_{x} \Phi^{s}\right)^{-1} F\left(\Phi^{s}(x)\right)
$$

converges to $F_{0}(x)$ uniformly on compact sets as $s \rightarrow-\infty$ $(s \rightarrow+\infty)$. Then $F_{0}$ is called the local $\nu$-homogeneous approximation of degree $d$ at $0($ at $+\infty)$ of $F$.

The local $\nu$-homogeneous approximation $F_{0}$ of degree $d$ is always homogeneous with degree $d$. Only one degree can give us an interesting approximation. An easy verification shows that, if for a given $d, F_{0}$ is non-vanishing, then all others approximations are either vanishing or not defined. When such a non-vanishing $\nu$-homogeneous approximation $F_{0}$ exists, we call it the $\nu$-homogenization of $F$. It is possible to show that if $F_{0}$ is the local $\nu$-homogeneous approximation of degree $d$ at 0 (at $+\infty)$ of $F$, then for any $\epsilon>0$ there is $s_{\epsilon}<0\left(s_{\epsilon}>0\right)$ such that

$$
\begin{gathered}
\sup _{y \in \mathbb{S}_{\nu}, \operatorname{sign}\left(s_{\epsilon}\right) s \geq\left|s_{\epsilon}\right|}|w(s, y)| \leq \epsilon, \\
w(s, y)=e^{-d s}\left(d_{y} \Phi^{s}\right)^{-1} F\left(\Phi^{s}(y)\right)-F_{0}(y),
\end{gathered}
$$

where $\mathbb{S}_{\nu}=\left\{x \in \mathbb{R}^{n}: N(x)=1\right\}$ and $N$ is a homogeneous norm. Further in this section the case of weighted homogeneity with $\nu(x)=\operatorname{diag}\left(r_{1} x_{1}, \ldots, r_{n} x_{n}\right)$ for some $r_{i} \in \mathbb{R}_{+}^{*}, 1 \leq i \leq n$ is considered (it is the most useful case for a local approximation calculation). In this case for any $x \in \mathbb{R}^{n}$ there exist $s \in \mathbb{R}$ and $y \in \mathbb{S}_{\nu}$ such that $x=\Phi^{s}(y)$ and

$$
\begin{aligned}
\left|F(x)-F_{0}(x)\right| & =\left|e^{d s} d_{y} \Phi^{s} w(s, y)\right| \\
& =\left|e^{d s} \operatorname{diag}\left(e^{r_{1} s}, \ldots, e^{r_{n} s}\right) w(s, y)\right| \\
& \leq \gamma(s)|w(s, y)|, \\
\gamma(s) & = \begin{cases}e^{\left(r_{\min }+d\right) s} & s \leq 0, \\
e^{\left(r_{\max }+d\right) s} & s>0,\end{cases}
\end{aligned}
$$

where $r_{\min }=\min _{1 \leq i \leq n} r_{i}$ and $r_{\max }=\max _{1 \leq i \leq n} r_{i}$. The following stability properties are satisfied Bacciotti and Rosier [2005], Zubov [1958], Andrieu et al. [2008]:

Theorem 17. Let $F$ be a continuous vector field and assume that there exists a non-zero vector field $F_{0}$ being its local approximation of degree $d$ at $0($ at $+\infty)$. If $F_{0}$ is
GAS, then $F$ is locally asymptotically stable (then $F$ has bounded trajectories).

The local approximation gives us a local information on the behavior of the system, it can be defined for any finite value $s_{0} \in(-\infty,+\infty)$ Efimov and Perruquetti [2010]. It can also be used to investigate the scale invariance property but locally. In the paper Skataric and Sontag [2012] it has been observed that the class of systems for which the scale invariance property holds exactly is very limited, and the notion of approximate scale invariance has been introduced.

Definition 18. For an $\varepsilon \in \mathbb{R}_{+}$, the system (2) is said to be $\varepsilon$-positive Approximately Scalable $(\varepsilon$-pAS) with respect to the inputs from $\mathbb{U}$ if there exists a function $\beta: \mathbb{R}_{+}^{*} \rightarrow \mathbb{R}_{+}^{*}$ such that for any $\bar{u} \in \mathbb{R}^{m}$, any input $u \in \mathbb{U}$ and any $\lambda>0$ :

$$
\sup _{t \geq 0}|y(t, \sigma(\lambda \bar{u}), \lambda u)-\beta(\lambda) y(t, \sigma(\bar{u}), u)| \leq \varepsilon .
$$

The system (2) is said to be logarithmic $\varepsilon$-pAS with respect to the inputs from $\mathbb{U}$ if for any $\bar{u} \in \mathbb{R}^{m}$, any input $u \in \mathbb{U}$ and any $\lambda>0$ there exists $\beta: \mathbb{R}_{+}^{*} \rightarrow \mathbb{R}_{+}^{*}$ such that

$$
\sup _{t \geq 0}\left|\frac{y(t, \sigma(\lambda \bar{u}), \lambda u)}{\beta(\lambda) y(t, \sigma(\bar{u}), u)}-1\right| \leq \varepsilon .
$$

The difference between $\varepsilon$-pAS and logarithmic $\varepsilon$-pAS is that the former one is better suited for analysis of approximate scalability at the origin (for small values of $|y|)$, while the latter property is better adapted for the scalability investigations for big values of $y$.

Obviously, if the system (2) is ISS with respect to the input $u$, then always there is an $\varepsilon>0$ (dependent on $u$ ) such that it is $\varepsilon$-pAS. The question is when this property is satisfied for a relatively small $\varepsilon$. An answer on this question has been given in Skataric and Sontag [2012] applying the time-separation and the linearization techniques. In this work we will use the local homogeneity framework for the same purpose. An advantage is that the local homogeneity allows an approximation at infinity (not only at the origin as with the linearization) to be defined.

\subsection{Relation of trajectories of $F$ and $F_{0}$}

First, let us try to establish the conditions under which the trajectories of a locally Lipschitz system

$$
\dot{x}=F(x),
$$

and its $\nu$-approximation

$$
\dot{x}=F_{0}(x),
$$

starting from the same initial conditions stay close. More precisely, denote $\Psi$ and $\Psi_{0}$ the flows of $F$ and $F_{0}$ respectively, select an $\varepsilon>0$, then we are looking for conditions that $\left|\Psi^{t}(x)-\Psi_{0}^{t}(x)\right| \leq \varepsilon$ for given $x \in \mathbb{R}^{n}$ for some time interval $t \in[0, T]$. Define $T_{x}^{r}=\left\{t \geq 0: \Psi^{t}(x) \in \mathcal{N}_{r}\right\}$, where $\mathcal{N}_{r}=\left\{x \in \mathbb{R}^{n}: \operatorname{sign}(r) N(x) \geq \operatorname{sign}(r) e^{r}\right\}$, note that the set $T_{x}^{r}$ can be empty if $\Psi^{t}(x) \notin \mathcal{N}_{r}$ for all $t \geq 0$. Lemma 19. Let $\varepsilon>0$ be given and $F_{0}$ be the local $\nu$ homogeneous approximation of degree $d$ at $0($ at $+\infty)$ of $F$. Let $F_{0}$ be a $C^{1}$ vector field and there exist matrices $\mathcal{A}_{k} \in \mathbb{R}^{n \times n}, 1 \leq k \leq K$ for some $K \in \mathbb{N}^{*}$ such that for all $x \in \mathbb{R}^{n}$

$$
\begin{gathered}
\operatorname{Jac} F_{0}(x) \in C o\left(\mathcal{A}_{1}, \ldots, \mathcal{A}_{K}\right), \\
\mathcal{A}_{k}^{T} P+P \mathcal{A}_{k}=-Q \quad \forall 1 \leq k \leq K
\end{gathered}
$$


for some matrices $P=P^{T}>0, Q=Q^{T}>0$. Then for all $x \in \mathcal{N}_{s_{\varepsilon / \rho}}$,

$$
\begin{gathered}
\left|\Psi^{t}(x)-\Psi_{0}^{t}(x)\right| \leq \varepsilon \kappa(x), \\
\kappa(x)=\max \left\{\xi \circ N(x)^{d+r_{\min }}, \xi \circ N(x)^{d+r_{\max }}\right\}
\end{gathered}
$$

while $t \in T_{x}^{s_{\varepsilon / \rho}}$, where $\rho=2 \sqrt{\frac{\lambda_{\max }^{3}(P)}{\lambda_{\min }^{2}(Q) \lambda_{\min }(P)}}$ and $\xi$ is a function from $\mathcal{K}_{\infty}$.

The condition that the Jacobian matrix of $F_{0}$ belongs to a convex hull of matrices (6) is widely used in the theory of convergent systems Pavlov et al. [2004] (together with the stability condition for all matrices in the hull) or for the incremental stability analysis Ingalls and Sontag [2002]. If $F_{0}$ is the local $\nu$-homogeneous approximation at 0 of $F$, then it is possible to select $\varepsilon>0$ sufficiently small in such a way that $\xi \circ N(x) \leq 1$, then the system has $\varepsilon$-pAS property while trajectories stay into $\mathcal{N}_{s_{\varepsilon / \rho}}$ if $d=0$. For the approximation at $+\infty$ the situation is more complicated, in this case decreasing the value of $\varepsilon$ implies increasing the norm of initial condition $N(x)$ for $x \in \mathcal{N}_{s_{\varepsilon / \rho}}$. However, if we would consider the coordinate ratio $\frac{\Psi_{i}^{t}(x)}{\Psi_{0, i}^{t}(x)}$ for any $1 \leq i \leq n$ under assumption that both variables $\Psi_{i}^{t}(x)$ and $\Psi_{0, i}^{t}(x)$ are separated from zero, then

$$
\left|\frac{\Psi_{i}^{t}(x)}{\Psi_{0, i}^{t}(x)}-1\right|=\left|\frac{\Psi_{i}^{t}(x)-\Psi_{0, i}^{t}(x)}{\Psi_{0, i}^{t}(x)}\right| .
$$

Next, let $d=0, \xi(s)=\xi_{0} s$ for some $\xi_{0}>0, r_{\max }=r_{\min }=$ 1 (these parameters correspond to a linear approximation dynamics $\left.F_{0}\right)$ and let $\Psi_{0, i}^{t}(x) \geq \eta N(x)$ for some $\eta>0$, then

$$
\left|\frac{\Psi_{i}^{t}(x)}{\Psi_{0, i}^{t}(x)}\right| \leq \varepsilon \frac{\xi_{0}}{\eta}
$$

for all $t \in T_{x}^{s_{\varepsilon / \rho}}$. In this case for a sufficiently small value of $\varepsilon$ the solutions of (4) and (5) stay close in "logarithmic" sense.

\subsection{Application of local homogeneity for $p A S$ analysis}

The main idea of this section is to combine the results of Theorem 12 and Lemma 19.

Take the system in (2) and an input $u \in \mathbb{U}$, which has an associated triple $\left(\phi, g, \xi_{0}\right)$. For the closed loop system (4) with $F$ defined in (3), let $F_{0}$ be $C^{1}$ local $\nu$ homogeneous approximation of degree 0 at $0($ at $+\infty)$ of $F$ for $\nu=\left(\begin{array}{ll}\nu_{1}^{T} & \nu_{2}^{T}\end{array}\right)^{T}$ for some Euler vector field $\nu_{1}$ (the vector field $g$ is $\nu_{2}$-homogeneous). In this case $F_{0}(x, \xi)=\left(f_{0}^{T}(x, \phi(\xi)) g^{T}(\xi)\right)^{T}$, where $f_{0}$ is a kind of local approximation of $f$, i.e.

$$
\left[d_{x} \Phi_{1}^{s}\right]^{-1} f\left(\Phi_{1}^{s}(x), \phi\left(\Phi_{2}^{s}(\xi)\right)\right) \stackrel{s \rightarrow \pm \infty}{\longrightarrow} f_{0}(x, \phi(\xi))
$$

uniformly on compact sets. Since all conditions of Theorem 12 are satisfied for $F_{0}$, it has the $\mathrm{pS}$ property. Intuitively, in the domain of $\mathbb{R}^{n}$ where $F$ and $F_{0}$ are sufficiently close, the original system (2) has pAS property for a properly selected $\varepsilon>0$. Indeed, as before denote $\Psi$ and $\Psi_{0}$ the flows of $F$ and $F_{0}$ respectively, then $p_{2} \circ \Psi^{t}=p_{2} \circ \Psi_{0}^{t}$. Define $e(t)=p_{1}\left(\Psi^{t}\left(x, \xi_{0}\right)-\Psi_{0}^{t}\left(x, \xi_{0}\right)\right)$, then

$$
\begin{aligned}
\dot{e}(t)= & f_{0}\left(p_{1} \circ \Psi^{t}, u(t)\right)-f_{0}\left(p_{1} \circ \Psi_{0}^{t}, u(t)\right) \\
& +f\left(p_{1} \circ \Psi^{t}, u(t)\right)-f_{0}\left(p_{1} \circ \Psi^{t}, u(t)\right) .
\end{aligned}
$$
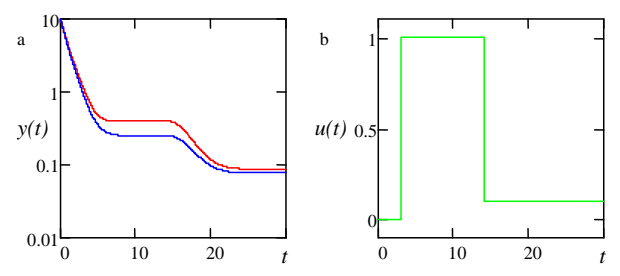

Fig. 1. The results of simulation for the system (7)

As in Lemma 19 , let $\varepsilon>0$ be given and there exist matrices $\mathcal{A}_{k} \in \mathbb{R}^{n \times n}, 1 \leq k \leq K$ for some $K \in \mathbb{N}^{*}$ such that for all $x \in \mathbb{R}^{n}$ and $u \in \mathbb{U}$

$$
\begin{aligned}
& \mathrm{Jac} f_{0}(x, u(t)) \in C o\left(\mathcal{A}_{1}, \ldots, \mathcal{A}_{K}\right), \\
& \mathcal{A}_{k}^{T} P+P \mathcal{A}_{k}=-Q \quad \forall 1 \leq k \leq K
\end{aligned}
$$

for some matrices $P=P^{T}>0, Q=Q^{T}>0$. Then for all $x \in \mathcal{N}_{s_{\varepsilon / \rho}}$, where $\rho$ is defined as in Lemma 19 ,

$$
\begin{gathered}
\left|\Psi^{t}\left(x, \xi_{0}\right)-\Psi_{0}^{t}\left(x, \xi_{0}\right)\right| \leq \varepsilon \kappa\left(x, \xi_{0}\right), \\
\kappa\left(x, \xi_{0}\right)=\max \left\{\xi \circ N\left(x, \xi_{0}\right)^{m+r_{\min }}, \xi \circ N\left(x, \xi_{0}\right)^{m+r_{\max }}\right\}
\end{gathered}
$$

while $t \in T_{x}^{s_{\varepsilon / \rho}}$. Therefore the system (2) has $2 \varepsilon \kappa\left(x, \xi_{0}\right)$ pAS property for $h(x)=x_{i}$ if approximation at the origin is considered or the logarithmic $2 \varepsilon \kappa\left(x, \xi_{0}\right)+\varepsilon^{2} \kappa^{2}\left(x, \xi_{0}\right)$ pAS property for the case of $\nu$-homogeneous approximation at $+\infty$. Let us consider examples of application.

Example 4. Consider a nonlinear cascade for some $n \in \mathbb{N}^{*}$ :

$$
\begin{aligned}
\dot{x}_{0} & =u-a_{0} x_{0}, \\
\dot{x}_{i} & =\frac{c_{i} x_{i-1}}{b_{i}+\left|x_{i-1}\right|}-a_{i} x_{i}, 1 \leq i \leq n, \\
y & =x_{n} .
\end{aligned}
$$

Let $\mathbb{U}$ be the class of piecewise constant inputs, then $g(\xi)=$ $0, \phi(\xi)=\xi$ and $\nu_{2}(\xi)=\xi$. Denote $x=\left(x_{0}, x_{1}, \ldots, x_{n}\right)^{T}$ and take $\nu_{1}(x)=x$, then the system has the local approximation of degree 0 at 0

$$
\begin{aligned}
\dot{x}_{0} & =\xi-a_{0} x_{0}, \\
\dot{x}_{i} & =\frac{c_{i}}{b_{i}} x_{i-1}-a_{i} x_{i}, 1 \leq i \leq n, \\
\dot{\xi} & =0
\end{aligned}
$$

and at $+\infty$

$$
\begin{aligned}
\dot{x}_{0} & =\xi-a_{0} x_{0}, \\
\dot{x}_{i} & =-a_{i} x_{i}, 1 \leq i \leq n, \\
\dot{\xi} & =0,
\end{aligned}
$$

which are stable linear systems (their stability follows the lower triangular structure of both). Thus the system (7) should demonstrate pAS property in almost all state space $\mathbb{R}^{n+1}$ for $p=1$ (linear scaling). The results of the system simulation for

$$
\begin{gathered}
n=2, a_{0}=a_{1}=a_{2}=1, c_{1}=c_{2}=1, \\
b_{1}=1, b_{2}=2,
\end{gathered}
$$

are shown in Fig. 1. The piecewise constant input $u(t)$ is presented in Fig. 1,b. The output trajectories $2 y(t)$ and $y(t)$ corresponding to the inputs $u(t)$ and $2 u(t)$ respectively are plotted in the logarithmic scale in Fig. 1,a.

Example 5. Following Ropers et al. [2009], consider a model of genetic regulatory network with a positive feedback: 

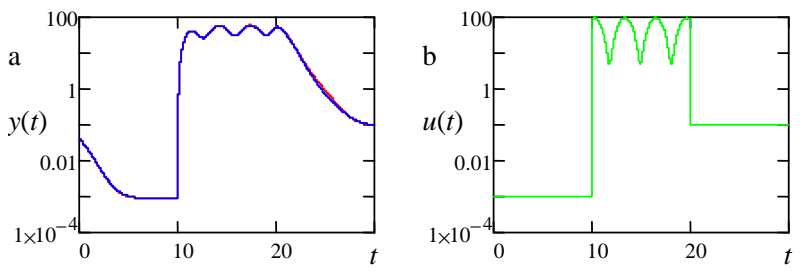

Fig. 2. The results of simulation for the system (8)

$$
\begin{aligned}
& \dot{x}_{1}=u(t)+\kappa_{1} \frac{x_{2}^{n}}{\theta_{1}+x_{2}^{n}}-\gamma_{1} x_{1}, \\
& \dot{x}_{2}=\kappa_{2} x_{1}-\gamma_{2} x_{2}, y=x_{2},
\end{aligned}
$$

where $x_{1} \in \mathbb{R}_{+}$and $x_{2} \in \mathbb{R}_{+}$are mRNA and protein concentrations respectively, $\kappa_{i}>0, \gamma_{i}>0$ are production and degradation rate constants respectively, $\theta_{1}>0, n>0$ are constant parameters, $u(t) \in \mathbb{R}_{+}$is external input modeled by a biased sinusoid of the frequency $\omega$. Take $\nu_{1}(x)=x$ and $\nu_{2}(\xi)=\xi$, then the system has the same local approximation of degree 0 at 0 and at $+\infty$

$$
\begin{aligned}
\dot{x}_{1} & =\xi_{1}+\xi_{2}-\gamma_{1} x_{1}, \\
\dot{x}_{i 2} & =\kappa_{2} x_{1}-\gamma_{2} x_{2}, \\
\dot{\xi_{1}} & =0, \\
\dot{\xi}_{2} & =\xi_{3}, \dot{\xi}_{3}=-\omega^{2} \xi_{2},
\end{aligned}
$$

which is a stable linear system (again, due to the lower triangular structure). Thus the system (8) should demonstrate pAS property in almost all state space $\mathbb{R}^{n+1}$ for $p=1$ (i.e. the trajectories of the system are scaled proportionally the input amplitude). The results of the system simulation for

$$
n=2, \kappa_{1}=\kappa_{2}=\theta_{1}=1, \gamma_{1}=\gamma_{2}=1.5, \omega=2
$$

are shown in Fig. 2. The piecewise continuous input $u(t)$ is presented in Fig. 2,b in the logarithmic scale. The output trajectories $2 y(t)$ and $y(t)$ corresponding to the inputs $u(t)$ and $2 u(t)$ respectively are plotted in the logarithmic scale in Fig. 2,a.

As we can conclude, these results of simulation confirm the theoretical development of the paper.

\section{CONCLUSION}

Local or global homogeneity of a vector field can be checked doing a simple calculus, thus verification of this property in practice is rather straightforward. In this work the relations between (approximate) scalability and (local) homogeneity are established. Some conditions on closeness of solutions of the original system and its local approximation are given. The theoretical results are confirmed by numerical experiments for models of biological cascades.

In the theory of homogeneity, it is well known that a system inherits stability/instability properties after its homogeneous approximation. The main result of this work claims that under mild additional conditions the solutions of the system and its homogeneous approximation stay close in the corresponding domains.

\section{REFERENCES}

U. Alon. An Introduction to Systems Biology: Design Principles of Biological Circuits. Chapman \& Hall, 2006.
V. Andrieu, L. Praly, and A. Astolfi. Homogeneous Approximation, Recursive Observer Design, and Output Feedback. SIAM J. Control Optimization, 47(4):18141850, 2008.

A. Bacciotti and L. Rosier. Lyapunov Functions and Stability in Control Theory. Springer, 2nd edition, 2005.

E. Bernuau, A. Polyakov, D. Efimov, and W. Perruquetti. ISS and iISS properties of homogeneous systems. In Proc. of ECC 2013, 2013a.

E. Bernuau, A. Polyakov, D. Efimov, and W. Perruquetti. Verification of ISS, iISS and IOSS properties applying weighted homogeneity. Systems \& Control Letters, 62 (12):1159-1167, 2013b.

E. Bernuau, D. Efimov, and W. Perruquetti. Robustness of homogeneous and locally homogeneous differential inclusions. In Proc. of ECC 2014, 2014.

S.P. Bhat and D.S. Bernstein. Geometric homogeneity with applications to finite-time stability. Mathematics of Control, Signals and Systems, 17:101-127, 2005.

D. Efimov and W. Perruquetti. Oscillations conditions in homogenous systems. In Proc. IFAC NOLCOS Symp., pages 1379-1384, 2010.

A.O. Hamadeh, B.P. Ingalls, and E.D. Sontag. Transient dynamic phenotypes as criteria for model discrimination: fold-change detection in rhodobacter sphaeroides chemotaxis. Proc. Royal Society Interface, 10:20120935, 2013.

H. Hermes. Nilpotent approximations of control systems and distributions. SIAM J. Control Optim., 24:731, 1986.

B. Ingalls and E.D. Sontag. A small-gain lemma with applications to input/output systems, incremental stability, detectability, and interconnections. J. Franklin Inst., 339:211-229, 2002.

M. Kawski. Geometric homogeneity and stabilization. In Arthur Krener and David Mayne, editors, Proc. IFAC Nonlinear Control Symposium, pages 164-169, Lake Tahoe, CA, 1995.

A. Pavlov, A. Pogromsky, N. van de Wouw, and H. Nijmeijer. Convergent dynamics, a tribute to boris pavlovich demidovich. Systems \& Control Letters, 52:257-261, 2004.

D. Ropers, H. de Jong, and J. Geiselmann. Systems Biology and Synthetic Biology, chapter Mathematical modeling of genetic regulatory networks : Stress responses in Escherichia coli, pages 235-271. John Wiley \& Sons, Hoboken, NJ, 2009.

L. Rosier. Homogeneous Lyapunov function for homogeneous continuous vector field. Systems \& Control Letters, 19:467-473, 1992.

O. Shoval, U. Alon, and E.D. Sontag. Symmetry invariance for adapting biological systems. SIAM Journal on Applied Dynamical Systems, 10:857-886, 2011.

M. Skataric and E.D. Sontag. A characterization of scale invariant responses in enzymatic networks. PLoS Computational Biology, 8:e1002748, 2012.

V.I. Zubov. On systems of ordinary differential equations with generalized homogenous right-hand sides. Izvestia vuzov. Mathematica., 1:80-88, 1958. in Russian. 